\title{
O Anunciado DSM-5: Que implicações em Psiquiatria Forense?
}

\author{
The Disclosed DSM-5: What impact will it have on Forensic Psychiatry?
}

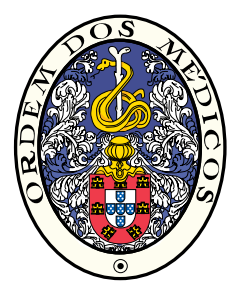

\author{
Susana FERNANDES ${ }^{1}$, Edna LEITE², Fernando VIEIRA ${ }^{3,5}$, Jorge COSTA SANTOS $3,4,5$ \\ Acta Med Port 2014 Jan-Feb;27(1):126-134
}

RESUMO

Introdução: Em 10 de Fevereiro de 2010, a American Psychiatric Association publicou online a versão preliminar (draft) da quinta edição do Manual de Diagnóstico e Estatística das Perturbações Mentais (Diagnostic and Statistical Manual of Mental Disorders), que, à semelhança das edições anteriores, depois de ter acolhido comentários e contribuições diversas, esteve sujeita, durante os dois anos seguintes, a um escrutínio técnico e científico, e, naturalmente, a alterações e revisões suscetíveis de conduzirem a uma versão final melhorada.

Material e Métodos: Este artigo visa analisar as alterações propostas por esta nova revisão, averiguar se estas têm implicações no domínio da Psiquiatria Forense e discutir as implicações identificadas.

Discussão: Nesta perspetiva, constituem objeto de análise as questões relacionadas com a classificação e estruturação das entidades nosológicas, com especial enfoque nas perturbações da personalidade, perturbações de abuso de substâncias, perturbações sexuais e da identidade de género, perturbações de ansiedade, perturbações dissociativas, perturbações psicóticas e, ainda, perturbações sugeridas por fontes externas.

Conclusão: As principais alterações propostas têm a ver essencialmente com os critérios de diagnóstico, que, relativamente a algumas das entidades nosológicas, poderão dificultar o resultado da avaliação pericial, enquanto em relação a outras poderá verificar-se o contrário, uma vez que a introdução de novos modelos de aproximação à realidade parece oferecer uma maior objetividade e rigor diagnósticos.

Palavras-chave: Manual de Diagnóstico e Estatística das Perturbações Mentais; Perturbações Mentais; Psiquiatria Forense; Portugal.

\section{ABSTRACT}

Introduction: On 10 February 2010, the American Psychiatric Association published an online draft of the fifth edition of the Diagnostic and Statistical Manual of Mental Disorders, which, like the previous editions, after having received several comments and contributions, was subject during the next following years, to the technical and scientific scrutiny, and, of course, susceptible to modification leading to a final improved version.

Material and Methods: This article aims to analyze the changes proposed by this new review, investigate whether these have implications in the field of Forensic Psychiatry and discuss the implications identified.

Discussion: In this perspective, the object of analysis are issues related to the classification and structuring of nosological entities, with special focus on personality disorders, substance abuse disorders, sexual and gender identity disorders, anxiety disorders, dissociative disorders, psychotic disorders and also disorders suggested by external sources.

Conclusion: the main changes proposed are essentially about diagnostic criteria, which, for some of nosological entities, may hinder the outcome of the expert assessment, while for others there could be otherwise, since the introduction of new models closer to reality appears to offer greater objectivity and diagnostic rigor.

Keywords: Diagnostic and Statistical Manual of Mental Disorders; Forensic Psychiatry; Mental Disorders; Portugal.

\section{INTRODUÇÃO}

Em 10 de Fevereiro de 2010, a American Psychiatric Association (APA) publicou no site http://www.dsm5.org a versão preliminar (draft) da quinta edição do Diagnostic and Statistical Manual of Mental Disorders (DSM).

Chefiados por David J. Kupfer, da Universidade de Pittsburg, e pelo doutor Darrel A. Regier, da APA, mais de 600 especialistas mundiais da saúde mental reúnem-se desde 1999 para debater os caminhos do diagnóstico psiquiátrico no século XXI. Os cientistas envolvidos foram agrupados tematicamente para oferecer recomendações nas seguintes áreas: nomenclatura, neurociência, desenvolvimento, prejuízo e incapacitação, problemas transcul- turais e lacunas no atual sistema de diagnóstico. A informação divulgada esteve disponível para escrutínio público entre 20 de Abril de 2010 e 15 de Junho de 2012. Durante este tempo, a APA realizou três fases de ensaios clínicos para testar alguns dos critérios de diagnóstico propostos no setting clínico, para avaliação da sua utilidade e aplicação prática. Prevê-se que a versão final do manual seja publicada em Maio de 2013. ${ }^{1}$ Os critérios propostos inscrevem-se, pois, num laborioso processo ainda em curso, pelo que têm caráter provisório.

O objetivo deste artigo é o de averiguar se as propostas contidas na agenda para o DSM-5 têm ou não relevân-

1. Departamento de Psiquiatria Vejle-Kolding. Serviços de Saúde Mental da Região Sul da Dinamarca. Vejle. Dinamarca.

2. Departamento de Psiquiatria. Hospital da Horta. Horta. Portugal.

3. Serviço de Clínica Forense. Delegação do Sul. Instituto Nacional de Medicina Legal e Ciências Forenses I.P. Lisboa. Portugal.

4. Faculdade de Medicina. Fundação para a Ciência e Tecnologia. Lisboa. Portugal.

5. CENCIFOR,Centro de Ciências Forenses. Faculdade de Ciências. Universidade de Lisboa. Lisboa. Portugal.

Recebido: 06 de Agosto de 2012 - Aceite: 26 de Fevereiro de 2013 | Copyright @ Ordem dos Médicos 2014 
cia para o exercício da Psiquiatria Forense, abordando as questões relacionadas com a classificação e estruturação, com particular enfoque nas categorias de diagnóstico de especial atinência pericial, como as perturbações da personalidade, as perturbações de abuso de substâncias, as perturbações sexuais e da identidade de género, as perturbações de ansiedade, as perturbações dissociativas, as perturbações psicóticas e, ainda, as perturbações sugeridas por fontes externas.

\section{MATERIAL E MÉTODOS \\ Modificações Estruturais}

Importa desde logo assinalar, pela relevância de que se reveste o objeto deste estudo, o facto de se encontrar prevista a alteração do atual sistema de classificação multiaxial, fundindo os eixos I, II e III num único eixo, que passará a conter todos os diagnósticos psiquiátricos e as 'condições médicas gerais'. Esta mudança configura uma aproximação ao sistema de classificação uniaxial da Classificação Internacional de Doenças (CID), da Organização Mundial de Saúde (OMS). Em relação aos eixos IV e V, estão previstas também algumas alterações, atualmente ainda em estudo. $^{2}$

\section{Perturbações da Personalidade}

A secção dedicada às perturbações da personalidade é, provavelmente, a que regista as mudanças mais significativas em todo o manual.

Em termos médico-legais, a alteração perspetivada de deslocar as perturbações da personalidade, atualmente incluídas no Eixo II, para o Eixo I, onde até agora têm figurado as doenças psiquiátricas propriamente ditas, poderá suscitar dúvidas quando da avaliação pericial dos pressupostos normativos da (in)imputabilidade. Dito de outro modo: fazer constar no mesmo eixo entidades nosológicas até aqui classificadas segundo uma lógica diversa poderá levar a admitir que o conhecimento científico tende a aproximar as perturbações da personalidade das doenças psiquiátricas, e que aquelas, nos termos da lei penal, podem constituir uma 'anomalia psíquica' geradora da inimputabilidade do autor de um crime.

Esta questão pode e deve ser equacionada, embora, em nosso entender, se trate de uma falsa questão. Com efeito, à luz da doutrina dominante no nosso país, as perturbações da personalidade devem ser incluídas - e não deixarão de o ser por isso - no conceito jurídico de 'anomalia psíquica', ainda que, na ausência de comorbilidade (tal como episódios psicóticos breves), não constituam, em regra, uma perturbação mental suficientemente grave que torne um indivíduo, no momento da prática de um facto, incapaz de avaliar a ilicitude deste ou de se determinar de acordo com essa avaliação, ou seja, que verifique os pressupostos normativos da inimputabilidade.

Aliás, quando os critérios e descrições textuais dos manuais de diagnóstico são utilizados com fins forenses, existe sempre um risco significativo de que a informação diagnóstica seja mal interpretada. Além disso, na maioria das situações, o diagnóstico clínico de uma perturbação mental à luz do DSM, não é suficiente para estabelecer a existência, para fins legais, de uma 'incapacidade mental' ou 'défice mental', conforme consta, aliás, no próprio manual DSM-IV. ${ }^{3}$

Em relação à concetualização das perturbações da personalidade, este draft propõe modificações significativas, que justificam uma breve descrição. O atual DSM-IV define perturbações da personalidade fundamentalmente por referência a défices em quatro áreas: pensamento, controlo de impulsos, afetividade e funcionamento interpessoal. ${ }^{3}$ Ao invés, o projeto de DSM-5 faz depender o conceito deste tipo de perturbação da existência de compromisso em apenas dois domínios: a identidade (sense of self) e o funcionamento interpessoal (relação com o outro). ${ }^{4}$

Em lugar dos atuais dez tipos de perturbações da personalidade, o DSM-5 pretende simplificar o sistema, reduzindo-as a apenas cinco: Antisocial/Psicopática, Evitante, Estado-limite (Borderline), Obsessiva-Compulsiva e Esquizotípica, eliminando, assim, as perturbações dos tipos Dependente, Histriónica, Narcísica, Paranóide e Esquizóide.

Adicionalmente, aponta-se para uma classificação do sujeito em função de seis domínios diferentes de traços de personalidade: emocionalidade negativa, introversão, antagonismo, desinibição, compulsividade e esquizotipia. Cada um destes domínios pode, por sua vez, ser subdividido em 4 a 10 traços de personalidade (trait facets), num total de 37 traços a serem classificados. O diagnóstico de uma perturbação da personalidade decorrerá, então, da existência de dificuldades significativas em uma ou ambas as áreas funcionais (identidade e relações interpessoais), as quais devem ser acompanhadas por pontuações elevadas nos domínios dos traços de personalidade; os défices deverão ocorrer durante um período de tempo significativo, não podendo ser explicados por outra perturbação ou pela utilização de substâncias. O clínico deverá pontuar, em cada sujeito, as dimensões dos traços de personalidade, do funcionamento e respetivo grau por referência à descrição de um protótipo.

Tomando por comparação o atual DSM-IV, a formulação diagnóstica do DSM-5 será realizada através da descrição prototípica da perturbação e não mediante a utilização de critérios de diagnóstico, desaparecendo a definição categorial de perturbação da personalidade. $\mathrm{O}$ diagnóstico deste tipo de perturbação torna-se, assim, mais difuso, podendo um indivíduo corresponder, em maior ou menor grau, a uma determinada perturbação consoante o seu grau de proximidade à constelação prototípica.

Esta nova concetualização da 'patologia da personalidade' poderá ter repercussões a nível da comunicação entre peritos médicos e juristas (magistrados e advogados). Por um lado, poderá acarretar dificuldades acrescidas quando, em sede de avaliação pericial, houver que explicar aos juristas a razão por que o autor de um ilícito penal, ainda que afetado por uma 'doença mental', verifica os pressupostos normativos da imputabilidade. Por outro lado, poderá facilitar a justificação de uma eventual diminuição da 
imputabilidade na medida em que o sistema de pontuações atrás referido permitirá aferir se o examinado corresponde ou não, e em que medida, àquela categoria diagnóstica.

Cabe aqui referir que este tipo de enquadramento diagnóstico, apesar de representar uma mudança desde há muito esperada na área das perturbações da personalidade,$^{5}$ poderá tornar a prática clínica psiquiátrica e pericial mais complexa e morosa.

\section{Perturbação de Uso de Substâncias}

Em relação a esta área, surgem duas alterações radicais: primeiro, a eliminação das categorias atualmen- te separadas de 'Abuso de Substâncias' e 'Dependência de Substâncias' e a sua substituição por uma categoria unificada, com a designação de 'Perturbação de Uso de Substâncias' (Substance-Use Disorder) - uma perturbação com gravidade clínica variável, cujo diagnóstico requer a verificação de apenas dois critérios (Tabela 1); segundo, a renomeação desta secção como 'Adição e Perturbações Relacionadas' (The Addiction and Related Disorders).

O termo 'dependência' foi considerado problemático pelos membros do grupo de trabalho dedicado a esta área, uma vez que se encontra associado à ideia de consumo de tóxicos de forma compulsiva e descontrolada. ${ }^{6}$ Operar

Tabela 1 - Critérios de diagnóstico para Perturbação de Uso de Substâncias, de acordo com a revisão proposta para o DSM-5*

\section{Perturbação de uso de substâncias}

Um padrão desadaptativo de utilização de substâncias conduzindo a um défice ou sofrimento clinicamente significativo, manifestado por dois (ou mais) dos seguintes, ocorrendo dentro de um período de 12 meses:

Utilização recorrente de uma substância, resultando na incapacidade de cumprir obrigações importantes no trabalho, na escola ou em casa (p. ex., ausências repetidas ou fraco desempenho profissional relacionados com a utilização de substâncias; ausências, suspensões ou expulsões escolares relacionadas com o uso da substância; negligência das crianças ou deveres domésticos)

Utilização recorrente da substância em situações em que tal se torna fisicamente perigoso (p. ex., conduzir um automóvel ou operar uma máquina quando afetado pela utilização da substância)

Uso continuado da substância, apesar dos problemas sociais ou interpessoais persistentes ou recorrentes, causados ou exacerbados pelos efeitos da substância (p. ex., discussões com o cônjuge sobre as consequências da intoxicação, agressões físicas)

Tolerância, definida por qualquer um dos seguintes:

a) Necessidade de quantidades crescentes da substância para obter a intoxicação ou efeito desejado

b) Diminuição acentuada do efeito com a utilização continuada da mesma quantidade de substância (Nota: A tolerância não é tida em conta em doentes que tomam medicamentos sob supervisão médica, como analgésicos, antidepressivos, ansiolíticos ou beta-bloqueantes)

5 Abstinência, definida por qualquer um dos seguintes:

a) Síndrome de abstinência característica da substância (ver os critérios A e B do conjunto de critérios para abstinência de substâncias específicas)

b) A mesma substância (ou outra estreitamente relacionada) é consumida para aliviar ou evitar sintomas (Nota: A tolerância não é tida em conta em doentes que tomam medicamentos sob supervisão médica, como analgésicos, antidepressivos, ansiolíticos ou beta-bloqueantes)

6 A substância é frequentemente consumida em quantidades superiores ou por um período mais longo do que o pretendido

7 Existe um desejo persistente ou esforços, sem sucesso, para abandonar ou controlar a utilização da substância

É despendido muito tempo em atividades necessárias à obtenção (p. ex., consultas a vários médicos ou deslocações) e utilização da substância ou à recuperação dos seus efeitos

É abandonada ou diminuída a participação em importantes atividades sociais, ocupacionais ou recreativas devido à utilização da substância

A utilização da substância é continuada, apesar da existência de um problema persistente ou recorrente, físico ou psicológico, provavelmente causado ou exacerbado pela utilização da substância

Especificadores de intensidade:

Moderada: 2-3 critérios positivos

Acentuada: 4 ou mais critérios positivos

(Fonte: http://www.dsm5.org/ProposedRevisions/Pages/proposedrevision.aspx?rid=431\#)

*A tradução é da responsabilidade dos autores. 
esta dissociação, procurando uma categoria diagnóstica mais abrangente e relativamente neutra, não representa, todavia, uma opção isenta de riscos. E isto porque a estigmatização que se pretendia evitar, poderá surgir por outra via, porventura até com alguma ressonância pejorativa, sobretudo quando utilizada para classificar indivíduos com consumos tóxicos intermitentes e temporários, muitas vezes influenciados por fatores de desenvolvimento e/ou contextuais. Com efeito, à luz da alternativa categorial proposta, estes indivíduos passarão a ser classificados como sofrendo de uma perturbação (doença) por uso de substâncias (de adição).

A fusão dos termos 'abuso' e 'dependência' afigura-se, assim, excessiva, porquanto, ignorando as diferenças de comportamento dos consumidores (ocasionais vs. frequen- tes/dependentes), não contempla as diferentes necessidades de tratamento e prognóstico de uns e de outros. ${ }^{7}$ Ora este aspeto tem manifestas implicações médico-legais, uma vez que passarão a ser incluídos na mesma categoria diagnóstica doentes com consumo esporádico de substâncias e outros delas dependentes (toxicodependentes), o que, à luz das disposições legais em vigor, poderá suscitar problemas não apenas no que respeita à avaliação pericial da (in)imputabilidade do autor de um crime, mas também em relação à prognose de 'perigosidade', ou seja, do risco de repetição de factos típicos idênticos. Por outro lado, se presentemente cabe ao perito médico fundamentar se o indivíduo é consumidor habitual, ou não, futuramente, e à luz dos critérios de diagnóstico DSM-5, o maior enfoque é diagnosticar o seu estado no momento em avaliação e

Tabela 2 - Critérios de diagnóstico para Perturbação de Hipersexualidade, de acordo com a revisão proposta para o DSM-59*

\section{Perturbação de Hipersexualidade}

Fantasias sexualmente excitantes, impulsos sexuais e comportamentos recorrentes e intensos durante um período de, pelo menos, seis meses, em associação com quatro ou mais dos seguintes cinco critérios:

(1) Consumo excessivo de tempo em fantasias, impulsos, planeamento e comportamento sexual

(2) Empreender repetidamente nestas fantasias, impulsos e comportamentos em resposta a humor disfórico

(p. ex., ansiedade, depressão, tédio, irritabilidade)

(3) Empreender repetidamente em fantasias, impulsos e comportamentos em resposta a eventos geradores de stresse

(4) Esforços repetitivos, mas sem sucesso, para controlar ou reduzir significativamente estas fantasias, impulsos e comportamentos

(5) Envolvimento repetido em comportamento sexual, menosprezando o risco de dano físico ou emocional para si próprio ou para outros

B A frequência e intensidade das fantasias, impulsos sexuais e comportamentos provocam mal-estar clinicamente significativo ou dificuldade no funcionamento social, ocupacional ou noutras áreas importantes

C As fantasias, impulsos sexuais e comportamentos não são devidos a efeitos fisiológicos diretos de substâncias exógenas (p. ex., drogas de abuso ou medicamentos) ou a Episódios Maníacos

D A pessoa tem pelo menos 18 anos de idade

Especificar se: $\quad$ Masturbação

Pornografia

Comportamento sexual consensual com adultos

Cibersexo

Sexo por telefone

Clubes de striptease

Outro:

Especificar se: Em remissão (Ausência de sinais e sintomas da perturbação durante os últimos seis meses) Em ambiente controlado

${ }^{*} \mathrm{~A}$ tradução é da responsabilidade dos autores

Tabela 3 - Critérios de diagnóstico para Perturbação Parafílica Coerciva, de acordo com a revisão proposta para o DSM-5^

\section{Perturbação Parafílica Coerciva}

A Fantasias sexualmente excitantes ou impulsos sexuais recorrentes e intensos, durante um período de, pelo menos, seis meses focados na coerção sexual.

B Os impulsos ou fantasias provocam mal-estar clinicamente significativo na pessoa ou esta tem procurado estimulação sexual através da coerção sexual de três ou mais pessoas em ocasiões distintas.

C O diagnóstico de Perturbação Parafílica Coerciva é excluído se o doente preenche critérios para um diagnóstico de Sadismo Sexual.

Especificar se: $\quad$ Em remissão (ausência de sinais ou sintomas da perturbação durante os últimos seis meses) Em ambiente controlado 
apenas nesse momento.

Mas são previsíveis outras - e porventura mais frequentes - repercussões em matéria de Direito de Família e Menores, nomeadamente em processos de regulação ou alteração do exercício das responsabilidades parentais, pois uma coisa será atribuir o poder paternal a um progenitor e não a outro, em virtude deste ser toxicodependente, e outra, bem diferente, preterir um dos pais em razão de um consumo ocasional - situações que agora surgem confundidas sob a mesma designação.

Impõe-se, então, que o perito psiquiatra se pronuncie não só em relação à categoria diagnóstica, mas que indique também os critérios que suportam a formulação diagnóstica de perturbação de uso de substâncias e os especificadores de gravidade, única forma de estabelecer a distinção entre os vários tipos de consumidores, aferir do seu maior ou menor grau de dependência e, consequentemente, das repercussões dos consumos nas respetivas esferas familiar, social, laboral e legal.

\section{Perturbações Sexuais e da Identidade de Género}

Nesta secção, preveem-se novas categorias diagnósticas que consideramos relevantes do ponto de vista forense: Perturbação de Hipersexualidade (Hypersexual Disorder - Tabela 2) e Perturbação Parafílica Coerciva (Paraphi- lic Coercive Disorder - Tabela 3) e a alteração de alguns dos critérios de diagnóstico de Pedofilia (Tabela 4).

A introdução de uma nova categoria diagnóstica denominada Perturbação de Hipersexualidade poderá ter sérias consequências forenses, nomeadamente na avaliação clínica de indivíduos acusados da prática de crimes de natureza sexual e na valoração da (in)imputabilidade, que assenta na capacidade de culpa e responsabilidade do agente. Do mesmo modo, o diagnóstico de Perturbação Parafílica Coerciva poderá ter também repercussões significativas nas avaliações pericial e judicial dos autores de crimes sexuais. Atualmente, uma das maiores dificuldades que se depara aos peritos médicos é a de estabelecer uma distinção clara e fiável entre o autor de um crime sexual relacionado com uma parafilia e a grande maioria dos abusadores sexuais, que obedece a outras motivações (p. ex., prazer, uso de poder, humilhação), ${ }^{8}$ visto existir o risco de uma eventual simulação, envolvendo mimetização sintomática, em indivíduos mais conhecedores do sistema de justiça e dos expedientes destinados a frustrar os seus desígnios. A vir a ser aprovada, tal categoria diagnóstica contribuirá, seguramente, para aumentar essas dificuldades.

Tendo em conta, por um lado, os factos mencionados nos argumentos da equipa de trabalho do DSM $-5,{ }^{9}$ de que

Tabela 4 - Critérios de diagnóstico para Pedohebefilia, de acordo com a revisão proposta para o DSM-5*

\section{Pedohebefilia}

A Existência, durante um período de, pelo menos 6 meses, de um ou ambos dos seguintes, implicando fantasias, impulsos ou comportamentos:

1. Excitação sexual recorrente e intensa por crianças na pré-puberdade ou na puberdade

2. Igual ou maior excitação por estas crianças do que por indivíduos fisicamente maduros

Um ou mais dos seguintes sinais ou sintomas:

1. A atração por crianças causa um mal-estar clinicamente significativo

2. A pessoa tem procurado estimulação sexual, em ocasiões distintas, de um dos seguintes:

a. Duas ou mais crianças diferentes, se ambas estão na pré-puberdade

b. Três ou mais crianças diferentes, se uma ou mais estão na puberdade

3. Utilização de pornografia representando crianças na pré-puberdade ou puberdade, preferindo-a a outra pornografia, por um período de, pelo menos, seis meses

C A pessoa tem no mínimo 18 anos e é pelo menos 5 anos mais velha que a criança ou crianças, tal como definidas nos critérios $A$ ou $B$

Especificar tipo:

Tipo Pedofilia - Atraído por crianças na pré-puberdade (geralmente com idade inferior a 11 anos)

Tipo Hebefilia - Atraído por crianças na puberdade (geralmente com idades entre os 11 e os 14 anos)

Tipo Pedohebefilia - Atração sexual por ambos

Especificar tipo:

Atraído por rapazes

Atraído por raparigas

Atraído por ambos

Especificar se:

Em remissão (ausência de sinais ou sintomas desta perturbação durante os últimos seis meses)

Em ambiente controlado

${ }^{\star} \mathrm{A}$ tradução é da responsabilidade dos autores 
a maioria dos violadores nega ter tido fantasias sexuais e, por outro, a dificuldade prática de realizar exames laboratoriais e a pouca fiabilidade destes, a formulação diagnóstica tenderá a basear-se fundamentalmente no relato do comportamento fornecido pelo próprio examinando, o que poderá traduzir-se por uma taxa elevada de falsos positivos. ${ }^{10}$

Por outro lado, é proposta a substituição do diagnóstico de Pedofilia pelo de Pedohebefilia (Pedohebephilic Disorder), com dilatação do limite etário, que anteriormente incluía apenas crianças pré-púberes, geralmente com 13 anos ou menos, ${ }^{10}$ passando agora a compreender também os adolescentes mais novos (até aos 14 anos), em consonância, aliás, com a categoria de Pedofilia, tal como esta se encontra definida na Classificação Internacional de Doenças (CID-10). ${ }^{11}$ É igualmente proposta a alteração do limite mínimo da idade do sujeito ativo, que no DSM-IV se encontrava fixado em 16 anos, passando, nesta nova edição, para os 18 anos de idade. Ambas as alterações têm repercussões não apenas na precisão do diagnóstico na prática clínica forense, mas também, porventura, em sede de inquérito judicial, uma vez que, à luz do nosso Código Penal, o crime de abuso sexual de crianças (artigo $171^{\circ}$ ) - um crime de natureza pública - refere-se a vítimas menores de 14 anos de idade.

\section{Esquizofrenia e Perturbações Psicóticas}

$\mathrm{Na}$ categoria diagnóstica que inclui a Esquizofrenia e outras Perturbações Psicóticas, o DSM-5 propõe uma revisão não apenas dos critérios de diagnóstico da doença, mas também dos subtipos até agora indicados para a Psicose Esquizofrénica.

Assim, são propostos como diagnósticos a incluir neste grupo de patologias a Síndrome de Sintomas Psicóticos Atenuados e um Especificador de Catatonia, sendo eliminados os subtipos E. Paranóide, E. Desorganizada (Hebefrénica), E. Catatónica, E. Indiferenciada, E. Residual e a Perturbação Psicótica Partilhada.

São, ainda, revistos os critérios de diagnóstico para a Esquizofrenia, a Perturbação Esquizofreniforme, a Perturbação Esquizoafetiva, a Perturbação Delirante e a Perturbação Psicótica Breve. Apenas não foram alvo de revisão a Perturbação Delirante devida a Condições Médicas e a Perturbação Psicótica SOE.

No âmbito deste trabalho, que tem por objeto a identificação e discussão de eventuais implicações forenses desta revisão do DSM, não poderia deixar de assinalar-se a relevância de propostas, tais como a inclusão da Síndrome de Sintomas Psicóticos Atenuados, a revisão dos critérios de diagnóstico e a eliminação dos subtipos diagnósticos.

\section{Síndrome de Sintomas Psicóticos Atenuados}

A eventual inclusão de uma categoria de diagnóstico que englobe um conjunto de sintomas psicóticos (conforme o proposto: ideias delirantes, alucinações, discurso desorganizado) de intensidade ligeira, mas persistente (pelo menos um mês no decurso de um ano) e não relacionado com outra patologia ou com o consumo de substâncias, parece razoável se tivermos em conta que muitos dos sintomas da esquizofrenia poderão surgir, de forma mais ou menos fruste, alguns anos antes de o diagnóstico ser colocado. Ora, vários estudos apontam justamente estes doentes como sendo aqueles que, frequentemente, consomem substâncias tóxicas, sobretudo canabinóides e álcool, numa tentativa de aliviarem sintomas geradores de mal-estar, e se envolvem em comportamentos delinquentes. Daí as implicações médico-legais.

No quadro clínico ora proposto, pode-se entrever a descrição do primeiro surto psicótico, entidade não categórica mas já identificada e reconhecida pela comunidade científica, onde se evidenciam de forma clara as alterações do pensamento e da perceção, que poderão abrir espaço à averiguação da (in)imputabilidade sempre que tal se justifique.

A investigação recente tem mostrado que uma intervenção terapêutica precoce condiciona um melhor prognóstico global, escorado numa resposta mais eficaz em termos cognitivos e/ou comportamentais, com rebate positivo na vida familiar, social e/ou profissional do paciente. Esta resposta terapêutica favorável vai contribuir, também, para reduzir o risco de comportamentos violentos e a repetição de ilícitos penais associados ao conceito jurídico de perigosidade.

\section{Revisão dos critérios de diagnóstico}

Foram revistos, uniformemente, os critérios de diagnóstico para a Esquizofrenia, Perturbação Esquizofreniforme, Perturbação Esquizoafetiva, Perturbação Delirante e Perturbação Psicótica Breve, tendo sido, ainda, introduzidas pequenas alterações no que concerne à Perturbação Esquizoafetiva (duração e intensidade dos sintomas afetivos), na tentativa de conferir uma maior consistência e fiabilidade a esta entidade nosológica.

A principal alteração diz respeito aos sintomas característicos destas perturbações, agrupados sob a designação 'critérios A' (Tabela 5).

Naquele que parece ser um distanciamento dos denominados sintomas de primeira linha de Schneider, deixa, assim, de ser permitido o diagnóstico quando está presente apenas um destes 'sintomas caraterísticos', desde que existam ideias delirantes bizarras ou alucinações comentadoras ou na terceira pessoa.

A dúvida poderá surgir perante indivíduos com sistemas delirantes complexos e bizarros, sem outras alterações, que irão ficar algures entre a Esquizofrenia e as Perturbações Delirantes ou, ainda, em relação a doentes que apresentem sobretudo alterações do comportamento (com isolamento social ou bizarrias), alterações motoras (catatónicas) e/ou sintomas negativos (com avolição, restrição dos afetos e defeito cognitivo), anteriormente passíveis de ser incluídos no diagnóstico de esquizofrenia enquanto subtipos - E. Catatónica, E. Simples, E. Residual ou E. Indiferenciada -, mas que agora deixam de ter lugar claro na classificação diagnóstica.

Esta é uma situação que, embora relativamente inócua 
Tabela 5 - Comparação (simplificada e traduzida) dos Critérios A de diagnóstico do DSM-5 vs. DSM-IV*

DSM-5 - Critérios A

(dois ou mais dos seguintes durante pelo menos 1 mês, ou menos se tratado, e pelo menos 1 destes deverá incluir um critério de 1 a 3)

1. Ideias delirantes

2. Alucinações

3. Discurso desorganizado

4. Catatonia

5. Sintomas negativos (afetos restritos ou avolição/isolamento social).

${ }^{\star} \mathrm{A}$ tradução é da responsabilidade dos autores

em relação à prática clínica, poderá revestir-se de especial relevância em sede de avaliação pericial psiquiátrica, nomeadamente se o perito for solicitado a pronunciar-se sobre os requisitos da (in)imputabilidade e da perigosidade. No caso da (in)imputabilidade, a questão é contornável, uma vez que se avalia a presença ou ausência de anomalia psíquica grave, baseada não em diagnósticos mas na existência, ou não, no momento da prática do facto, da capacidade do sujeito para avaliar a ilicitude do facto cometido e da liberdade para se determinar de acordo com essa avaliação. Quanto à perigosidade, entendida como a probabilidade de repetição do facto ilícito típico da mesma espécie, esta deverá ter em conta os dados estatísticos e as probabilidades associadas às patologias identificadas, cenário que poderá encerrar alguma dificuldade quando o diagnóstico oferecer dúvidas ou não existirem dados estatísticos que contemplem este novo enquadramento.

É curioso verificar que a investigação realizada até à data mostrou uma associação inequívoca entre comportamento violento e perturbação mental, em particular com a E. Paranóide, havendo abundantes referências a quadros clínicos caraterizados pela existência de sintomas positivos bem sistematizados e particularmente intensos. Essas referências vêm, afinal, reforçar algo que releva da lógica mais elementar: a presença de sintomatologia produtiva, sobretudo a de cariz paranóide persecutório, confere um maior risco e predisposição para o comportamento violento.

Assim, não é por desaparecerem do manual de classificação os subtipos atrás referidos que os procedimentos diagnósticos e outros relativos à (in)imputabilidade do sujeito deixarão de ser efetuados, até porque esta questão se inscreve, segundo as disposições legais em vigor, no conceito mais amplo de 'anomalia psíquica', exigindo, além disso, a verificação de outros requisitos, como a capacidade ou incapacidade do agente para, em um dado momento, avaliar a ilicitude do facto ou se determinar de acordo com essa avaliação. Algo paradoxalmente, esta alteração na categorização, pondo termo a esses subtipos, poderá fazer apelo a uma maior concentração do perito no indivíduo doente e na respetiva sintomatologia, e, consequentemente, na obrigatoriedade de uma mais ampla e rigorosa
DSM IV - Critérios A

(dois ou mais dos seguintes durante pelo menos 1 mês, ou menos se tratado)

1. Ideias delirantes

2. Alucinações

3. Discurso desorganizado

4. Comportamento marcadamente desorganizado ou catatónico

5. Sintomas negativos (afectos embotados, alogia ou avolição)

descrição contextual. Será este um grande desafio da Psiquiatria Forense: maior exigência nas descrições, uma vez que o número de classificações irá ser reduzido. Exigir-se-á maior conhecimento do essencial neste domínio para meIhor poder colaborar na administração da Justiça.

\section{Perturbações Dissociativas}

No que se refere às Perturbações Dissociativas, a nova revisão vem propor que a Fuga Dissociativa e a Perturbação de Despersonalização passem a constituir, respetivamente, subtipos da Amnésia Dissociativa e da Perturbação Dissociativa da Identidade.

Especificamente em relação ao diagnóstico de Fuga Dissociativa, o argumento invocado é que a alteração primária é da memória, habitualmente sob a forma de amnésia dissociativa quanto à identidade, enquanto a fuga surge raramente e de forma pouco consistente, pelo que faz todo o sentido que esta constitua apenas uma subcategoria do diagnóstico primário - Amnésia Dissociativa.

As perturbações dissociativas, sob o ponto de vista jurídico, podem ser incluídas no conceito de 'doença psiquiátrica transitória', um conceito bastante genérico, de reverberação americanizada, que se refere, afinal, a uma anomalia psíquica de ocorrência muito rara, passageira, particularmente intensa e potencialmente recorrente. Em nosso entender, a relevância deste tipo de perturbações poderá situar-se sobretudo a nível do $\mathrm{n}^{\circ} 2$ do artigo $20^{\circ}$ do Código Penal para justificar a imputabilidade sensivelmente diminuída. O benefício deste novo arranjo diagnóstico residirá, porventura, na definição de critérios mais estritos e, portanto, num maior rigor diagnóstico e numa mais correta aplicação da justiça.

\section{Perturbações de Ansiedade}

Para a classificação diagnóstica que descreve as Perturbações de Ansiedade, o DSM-5 propõe a eliminação e reagrupamento de alguns subtipos categoriais e a revisão de critérios diagnósticos. Veja-se, desde logo, as alterações sugeridas para a Perturbação Pós-Stresse Traumático (PPST). Estas modificações visam sobretudo uma maior fiabilidade do diagnóstico, especificando as situações potencialmente traumáticas, caracterizando mais exaustiva- 
mente o quadro clínico típico (dividido agora em alterações negativas do humor e da cognição) e introduzindo alguns termos menos abrangentes de molde a reduzir as possibilidades diagnósticas.

Em sede de Direito Civil está prevista a reparação do dano psíquico sofrido em consequência de um acontecimento especialmente traumático, direto ou indireto, que tenha irrompido de forma brutal na vida de um indivíduo, excedendo manifestamente a sua capacidade de integração. É sobretudo neste contexto que é suscitada uma avaliação pericial, visando estabelecer se o examinando apresenta uma perturbação mental (PPST ou outra), o nexo de causalidade entre o dano e o evento traumático, as consequências do dano (temporárias e permanentes) para a vida do sujeito e as possibilidades de recuperação deste.

Naturalmente, esta é sempre uma avaliação que envolve uma considerável subjetividade, pelo que a definição de critérios de diagnóstico mais estritos não deixará de contribuir para um resultado pericial mais seguro e rigoroso.

\section{Perturbações propostas por fontes externas}

Diversas fontes externas, tais como grupos e associações com intervenção na área da saúde mental, têm vindo a propor a inclusão no DSM-5 de uma série de outras perturbações. Estas propostas encontram-se, de momento, em fase de análise pelos grupos de trabalho constituídos, não havendo, ainda, elementos que permitam antecipar, com razoável segurança, quais dessas propostas virão a ter acolhimento na futura edição do manual.

Uma das perturbações, ainda em fase de análise, é a designada Síndrome de Alienação Parental (SAP). Trata-se de um conceito introduzido por Richard Gardner, em 1985, relacionado com situações em que um dos progenitores procura subtrair ao outro um filho de ambos, levando a cabo uma série de ações intencionais, sistemáticas, mais ou menos elaboradas e continuadas no tempo, visando concretizar esse objetivo. É uma disfunção do vínculo afetivo parental, que envolve a transformação de um vínculo positivo em negativo. ${ }^{12}$

Trata-se de um constructo operacional, de natureza mais sociológica do que clínica e que, até à data, não corresponde a qualquer doença ou entidade nosológica médica reconhecida pela comunidade científica e acolhida nos sistemas de classificação internacionais. Todavia, no nosso país, tem-se vindo a registar um número crescente de pedidos de avaliação pericial por parte dos Tribunais de Família e Menores, cujo objeto é, precisamente, a averiguação de uma alegada Síndrome de Alienação Parental. Ora, a vir a ser acolhido como entidade nosológica no DSM-5, o diagnóstico psiquiátrico de SAP não poderá deixar de afetar a credibilidade do progenitor alienado, com as consequentes repercussões nos processos judiciais de regulação do exercício das responsabilidades parentais.

\section{DISCUSSÃO}

O objetivo visado não era fazer uma revisão exaustiva do tema, mas tão só uma reflexão sobre eventuais repercussões que a nova classificação nosológica, tal como figura na agenda do DSM-5, da American Psychiatric Association, poderá ter no exercício da psiquiatria forense e, por extensão, na realização da Justiça em Portugal. O enfoque foi, sobretudo, na vertente do direito penal, por ser esta que, à luz do ordenamento jurídico interno, mais questões suscita. Ainda assim, não deixou de se fazer referência a uma entidade nosológica - a Perturbação Pós-Stresse Traumático -, dada a importância de que esta se reveste em matéria de direito civil e direito do trabalho.

As principais alterações propostas têm a ver, como se disse, com os critérios de diagnóstico, que, relativamente a algumas das entidades nosológicas, poderão dificultar, pela sua rigidez, o resultado da avaliação pericial, enquanto em relação a outras poderá verificar-se o contrário, uma vez que a introdução de novos modelos de aproximação à realidade parece oferecer uma maior objetividade e rigor diagnósticos.

Porém, não nos iludamos. Algo poderá ainda mudar até à publicação do DSM-5, cuja versão final, aprovada pelo Conselho, foi, entretanto, submetida ao American Psychiatric Publishing.

\section{CONCLUSÕES}

A reunião anual da APA, em São Francisco, de 18 a 22 de maio de 2013, marcará o lançamento oficial do DSM-5, com tudo o que isto representa em termos de dificuldade de adaptação a uma realidade, que se anuncia como substancialmente diferente, porventura mesmo de rutura paradigmática. E a experiência ensina-nos que as mudanças radicais obrigam a uma rutura com práticas há muito enraizadas, acarretando esforços acrescidos. No caso presente, não apenas aos psiquiatras em geral e aos que - e são quase todos - exercem funções periciais, mas também aos juristas, e sobretudo aos magistrados, que se movem num quadro epistemológico diverso. Uns e outros devem estar pelo menos alertados para uma nova realidade, ainda que em gestação, porque esta não deixará de afetar o seu quadro de referências teóricas e as suas práticas profissionais.

\section{CONFLITOS DE INTERESSE}

Os autores declaram a inexistência de conflitos de interesse na realização deste trabalho.

\section{FONTES DE FINANCIAMENTO}

Os autores declaram a inexistência de fontes de financiamento externas. 


\section{REFERÊNCIAS}

1. Yan, J. APA Makes DSM-5 proposals available for comment. Psychiatric News. [consultado 2010 jun 20]. Disponível em: http:// pn.psychiatryonline.org/content/45/5/1.1.full.

2. American Psychiatric Association: DSM 5 development - DSM-5 overview: the future manual. [consultado 2010 jun 20]. Disponível em: http:// www.dsm5.org/ProposedRevisions/Pages/ClassificationlssuesUnderDiscussion.aspx.

3. American Psychiatric Association. DSM-IV-TR: Manual de diagnóstico e estatística das perturbações mentais. Lisboa: Climepsi Editores; 2002.

4. American Psychiatric Association: DSM 5 development - personality and personality disorders. [consultado 2010 Jun 20]. Disponível em: http://www.dsm5.org/ProposedRevisions/Pages/PersonalityandPersonalityDisorders.aspx.

5. DeFife, J. DSM-V offers new criteria for personality disorders. Psychology Today. [consultado 2010 Jun 20]. Disponível em: http://www.psychologytoday.com/blog/the-shrink-tank/201002/dsm-v-offers-new-criteria-personality-disorders.

6. American Psychiatric Association: DSM 5 Development - Substance Related Disorders. [consultado 2010 Jun 20]. Disponível em: http:// www.dsm5.org/ProposedRevisions/Pages/Substance-RelatedDisor- ders.aspx.

7. Frances A. DSM 5 "Addiction" Swallows Substance Abuse. Psychology Today. [consultado 2010 Jun 20]. Disponível em: http://www.psychologytoday.com/blog/dsm5-in-distress/201003/dsm5-addiction-swallows-substance-abuse.

8. Frances A. Opening Pandora's box: The 19 worst suggestions for DSM 5. 2010. [consultado 2010 Jun 20]. Disponível em: http://www.psychiatrictimes.com/dsm-v/content/article/10168/1522341.

9. American Psychiatric Association: DSM 5 development - proposed revisions. [consultado 2010 Jun 20]. Disponível em: http://www.dsm5.org/ ProposedRevisions/Pages/proposedrevision.aspx?rid=416\#.

10. Frances A, Sreenivasan S, Weinberger LE. Defining mental disorder when it really counts - DSM-IV-TR and SVP/SDP statutes. J Am Acad Psychiatry Law. 2008;36:375-84.

11. American Psychiatric Association: DSM 5 development - pedophilia [consultado 2010 Jun 20]. Disponível em: http://www.dsm5.org/ProposedRevisions/Pages/proposedrevision.aspx?rid=186\#.

12. Cintra P, Salavessa M, Pereira B, Jorge M, Vieira F. Síndroma de alienação parental: realidade médico-psicológica ou jurídica? Rev Julgar. 2009;7:197-205. 


\section{O Anunciado DSM-5: Que implicações em Psiquiatria Forense? \\ Acta Med Port 2014:27:126-134}

Publicado pela Acta Médica Portuguesa, a Revista Científica da Ordem dos Médicos

Av. Almirante Gago Coutinho, 151

1749-084 Lisboa, Portugal.

Tel: +351218428 215

E-mail: submissao@actamedicaportuguesa.com

www.actamedicaportuguesa.com

ISSN:0870-399X | e-ISSN: 1646-0758

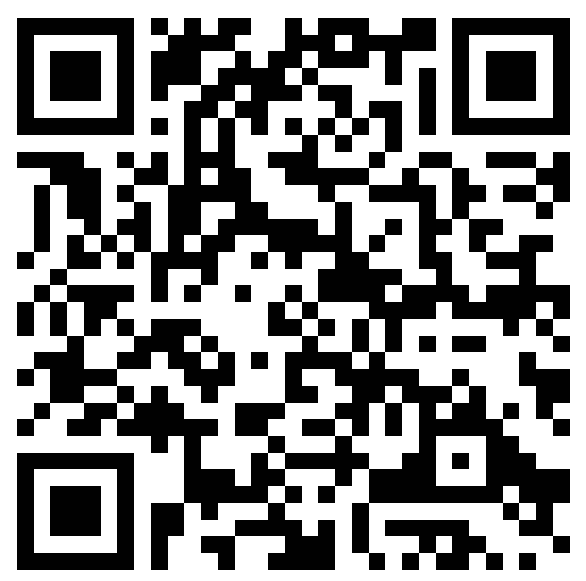

